\title{
EXAMINATION THE ABC CONJECTURE USING SOME FUNCTIONS.
}

\author{
SEIJI Fujino ${ }^{\dagger} 1$
}

2022.Jan.31

\begin{abstract}
In this paper, we discuss the negative consequence of the weak abc conjecture. Namely, we derive that the weak abc conjecture is not true. Additionally, we show that the strong abc conjecture is not true.
\end{abstract}

\section{The ABC Conjecture.}

\subsection{Introduction.}

The purpose of this paper is to derive that the negative consequence of the weak abc conjecture is true. Namely, we show that the weak abc conjecture is not true. Besides, we show that the strong abc conjecture is not true.

The proof steps are as follows :

1.1.1. First, we define the function $R_{\alpha}^{-}(x)$ as follows :

$$
R_{\alpha}^{-}(x):=\frac{\sqrt{2 \pi} \alpha}{e x-1}
$$

where variables $x$ and $\alpha$ are positive real numbers.

We further defined as follows : Let $\epsilon>0$ be positive real numbers and $K_{\epsilon}>0$ be the constant. For all positive integer $a>1, b>1$ and $c>0$ such that $a+b=c$ the function $P_{K_{\epsilon}}(a, b, c)$ is defined as follows:

$$
P_{K_{\epsilon}}(a, b, c):=\frac{\log (c)}{\log \left(K_{\epsilon} \operatorname{rad}(a b c)\right)} .
$$

1.1.2. Second, we prove that $P_{K_{\epsilon}}(a, b, c)>1$ and $P_{K_{\epsilon}}(a, b, c)$ is monotonically increasing. Furthermore we show that the following theorem is satisfied. Namely,

Theorem. (Below theorem(1.7)).

Let $\alpha>0$ be a positive real number. For all real number $\epsilon>0$ and constant $K_{\epsilon} \geq 1$, there exists countable infinite triples $(a, b, c)$ of coprime positive integers with $a+b=c$ such that the following inequality is satisfied:

$$
K_{\epsilon} \operatorname{rad}(a b c)<c^{\exp \left(\frac{e}{\sqrt{2 \pi} \alpha}\right)-1} .
$$

1991 Mathematics Subject Classification. Primary 11D04; Secondary 11D41.

Key words and phrases. Number theory, Diophantine equation, abc conjecture.

$1_{\dagger}$ RHC institute. No.702, Shinjuku-Komuro-BLD.,4-1-22 Shinjuku,Shinjuku-ku,Tokyo,Japan. 160-0022,xfujino001@gmail.com,xfujino001@rhc-institute.com 
1.1.3. Third, using these result above, we derive that the negative consequence of the weak abc conjecture is true. Namely, we prove that the weak abc conjecture is not true. Moreover, we prove that the strong abc conjecture is not true.

\subsection{The weak abc conjecture.}

We first describe the definitions and the weak abc conjecture.

Definition 1.1. Let $a, b, c \in \mathbb{N}$ be positive integers :

$$
\begin{aligned}
& (a, b, c): \text { the triples of coprime positive integers. } \\
& \operatorname{rad}(a b c)=\prod_{p \mid a b c, p: \text { prime }} p
\end{aligned}
$$

Conjecture 1.2. The weak abc conjecture.

For all positive real number $\epsilon>0$, there exists only finite many triples $(a, b, c)$ of coprime positive integers with $a+b=c$ such that the following inequality is satisfied

$$
\operatorname{rad}(a b c)^{1+\epsilon}<c
$$

This formulation is equivalent to the following conditions :

For all positive real number $\epsilon>0$, there exist a constant $K_{\epsilon} \geq 1$ such that for all triples $(a, b, c)$ of coprime positive integers with $a+b=c$, such that the following inequality is satisfied:

$$
c<K_{\epsilon} \operatorname{rad}(a b c)^{1+\epsilon} .
$$

Next, we show that the well-known fact $\operatorname{rad}(a b c)<c$ as follows.

Lemma 1.3. Example of $\operatorname{rad}(a b c)<c$.

Let $n>0$ be a positive integer. The triples $(a, b, c)$ of coprime integers $a=1, b=$ $3^{2^{n}}-1, c=3^{2^{n}}$ with $a+b=c$ such that the following inequality satisfied :

$$
\operatorname{rad}(a b c)<c=3^{2^{n}} .
$$

Namely, There exists infinite triples $(a, b, c)$ of coprime integers such that satisfies as follows:

$$
\operatorname{rad}(a b c)<c .
$$

Proof. The proof is described in the following section.

We define the function $P_{K_{\epsilon}}(a, b, c)$ and $R_{\alpha}^{-}(x)$ as follows.

Definition 1.4. Let $\epsilon>0$ be positive real numbers and $K_{\epsilon}>0$ be the constant. For all positive integer $a>1, b>1$ and $c>1$ such that $a+b=c$ the function $P_{K_{\epsilon}}(a, b, c)$ is defined as follows:

$$
P_{K_{\epsilon}}(a, b, c):=\frac{\log (c)}{\log \left(K_{\epsilon} \operatorname{rad}(a b c)\right)}
$$


Definition 1.5. Let $\alpha>0$ be a positive real number $(\in \mathbb{R})$. For all positive real number $x>1(\in \mathbb{R})$, the functions $R_{\alpha}^{-}(x)$ is defined as follows:

$$
R_{\alpha}^{-}(x):=\frac{\sqrt{2 \pi} \alpha}{e x-1}
$$

Therefore, the following equation is satisfied:

$$
\frac{1}{x R_{\alpha}^{-}(x)}=\frac{e}{\sqrt{2 \pi} \alpha}\left(1-\frac{1}{e x}\right) .
$$

Lemma 1.6. Let $a, b, c$ and $n$ be satisfied the condition on Lemma(1.3). Namely, put $a=1, b=3^{2^{n}}-1, c=3^{2^{n}}$ where $n>0$ is a positive integer. For all positive real number $\epsilon>0, K_{\epsilon}>0$ and sufficiently large positive integer $n>0$ the following conditions are satisfied:

$$
\begin{aligned}
& P_{K_{\epsilon}}(a, b, c)=\frac{\log (c)}{\log \left(K_{\epsilon} \operatorname{rad}(a b c)\right)}>1 . \\
& P_{K_{\epsilon}}(a, b, c) \text { is monotonically increasing. }
\end{aligned}
$$

Proof. The proof is described in the following section.

Theorem 1.7. Let $\alpha>0$ be a positive real number. For all real number $\epsilon>0$ and constant $K_{\epsilon} \geq 1$, there exists countable infinite triples $(a, b, c)$ of coprime positive integers with $a+b=c$ such that the following inequality is satisfied:

$$
K_{\epsilon} \operatorname{rad}(a b c)<c^{\exp \left(\frac{e}{\sqrt{2 \pi} \alpha}\right)-1}
$$

Proof. The proof is described in the following section.

Set $\alpha$ in Theorem(1.7) as follow:

$$
\alpha=\frac{e}{\sqrt{2 \pi} \log \left(\frac{\epsilon+2}{\epsilon+1}\right)} .
$$

Namely, the exponet of $c$ on the inequality ( 1.15 ) in theorem(1.7) set as follows:

$$
\frac{1}{1+\epsilon}=\exp \left(\frac{e}{\sqrt{2 \pi} \alpha}\right)-1 \text {. }
$$

Consequently, the negative consequence of the weak abc conjecture is satisfied as follows :

Theorem 1.8. The negation of the weak abc conjecture.

For all real number $\epsilon>0$ and constant $\bar{K}_{\epsilon} \geq 1$, there exists countable infinite triples $(a, b, c)$ of coprime positive integers with $a+b=c$ such that the following inequality is satisfied:

$$
\bar{K}_{\epsilon} \operatorname{rad}(a b c)^{1+\epsilon}<c .
$$

Namely, There is a counter-example in the weak abc conjecture. Therefore, the weak abc conjecture is not true.

Proof. The proof is described in the following section. 


\subsection{The strong abc conjecture.}

Next, we also examine the strong abc conjecture as follows :

Conjecture 1.9. The strong abc conjecture.

For all triples $(a, b, c)$ of coprime positive integers with $a+b=c$ such that the following inequality is satisfied:

$$
c<\operatorname{rad}(a b c)^{2}
$$

Using Theorem(1.8), that is, the weak abc conjecture is not true, we obtain the following result :

Theorem 1.10. The negation result of the strong abc conjecture.

There exists countable infinite triples $(a, b, c)$ of coprime positive integers with $a+$ $b=c$ such that the following inequality is satisfied:

$$
\operatorname{rad}(a b c)^{2}<c
$$

Namely, the strong abc conjecture is not true.

Proof. By theorem(1.8), put $\epsilon=1$ and $\bar{K}_{\epsilon}=1$, the following conditions are satisfied:

$$
\operatorname{rad}(a b c)<c^{\frac{1}{2}}
$$

Triples of coprime $a=1, b=3^{2^{n}}-1$, and $c=3^{2^{n}}$ are satisfied $a+b=c$, Therefore the following conditions are satisfied :

$$
\operatorname{rad}(a b c)^{2}<c \quad\left(\epsilon=1, \bar{K}_{\epsilon}=1\right) .
$$

After all, There exists countable infinite triples $(a, b, c)$ of coprime integers such that satisfies the strong abc conjecture. Namely, There is a counter-example in the strong abc conjecture. Therefore, the strong abc conjecture is not true.

\section{Proof of the ABove Discussion.}

We give proofs of the above section. We fisrt give the proof of Lemma(1.3), second Lemma(1.6) and third Theorem(1.7) as follows.

\subsection{Proof of Lemma(1.3).}

Proof. The following condition are satisfied :

$$
3^{2^{n}}=9^{2^{n-1}} \equiv 1(\bmod 8) .
$$

Thus,

$$
b=3^{2^{n}}-1 \text { is divisible } 8 .
$$

Hence, the following condition is satisfied :

$$
\operatorname{rad}\left(3^{2^{n}}-1\right)<\frac{1}{4} \operatorname{rad}\left(3^{2^{n}}-1\right)
$$


Therefore,

$$
\begin{aligned}
\operatorname{rad}(a b c) & \leq \operatorname{rad}(a) \operatorname{rad}(b) \operatorname{rad}(c) \\
& \leq \frac{1}{4} b \cdot 3 \quad(\because(2.3) \text { and } \operatorname{rad}(\mathrm{c})=3) \\
& <\frac{3}{4} c \quad(\because b<c) \\
& <c .
\end{aligned}
$$

\subsection{Proof of Lemma(1.6).}

Proof. The functions $f$ and $g$ are defined as follows:

$$
\begin{aligned}
& f(n):=\log \left(3^{2^{n+1}}\right)-\log \left(3^{2^{n}}\right) . \\
& g(n):=\log \left(K_{\epsilon} \operatorname{rad}\left(3^{2^{n+1}}\left(3^{2^{n+1}}-1\right)\right)\right)-\log \left(K_{\epsilon} \operatorname{rad}\left(3^{2^{n}}\left(3^{2^{n}}-1\right)\right)\right) .
\end{aligned}
$$

Derive that for all $n>1$ the relation of functions $f(n)$ and $g(n)$ are satisfied as follows :

$$
f(n)>g(n) .
$$

First, the following inequality are satisfied :

$$
\begin{aligned}
3^{2^{n}} & =\frac{3^{2^{n}} 3^{2^{n}}}{3^{2^{n}}} \\
& =\frac{3^{2^{n+1}}}{3^{2^{n}}} \\
& >\frac{\operatorname{rad}\left(3^{2^{n+1}}\left(3^{2^{n+1}}-1\right)\right)}{\operatorname{rad}\left(3^{2^{n}}\left(3^{2^{n}}-1\right)\right)} \\
& =\frac{K_{\epsilon} \operatorname{rad}\left(3^{2^{n+1}}\left(3^{2^{n+1}}-1\right)\right)}{K_{\epsilon} \operatorname{rad}\left(3^{2^{n}}\left(3^{2^{n}}-1\right)\right)}
\end{aligned}
$$

Hence, for all integer $n>1$, the following inequalities are satisfied :

$$
\begin{aligned}
f(n) & =\log \left(3^{2^{n+1}}\right)-\log \left(3^{2^{n}}\right) \\
& >\log \left(K_{\epsilon} \operatorname{rad}\left(3^{2^{n+1}}\left(3^{2^{n+1}}-1\right)\right)\right)-\log \left(K_{\epsilon} \operatorname{rad}\left(3^{2^{n}}\left(3^{2^{n}}-1\right)\right)\right) \\
& =g(n)
\end{aligned}
$$

Therefore, for all integer $n>0$, the relation of the functions $f(n)$ and $g(n)$ are satisfied :

$$
f(n)>g(n) .
$$

Because the inequality $f(n)>g(n)$ is satisfied, therefore the rate of increase in the numerator of $P_{K_{\epsilon}}(a, b, c)$ is greater than the rate of increase in the denominator of $P_{K_{\epsilon}}(a, b, c)$. Consequently, $P_{K_{\epsilon}}(a, b, c)$ is monotonically increasing.

Here, assume that $P_{K_{\epsilon}}(a, b, c)$ is not monotonically increasing. (reductio ad absurdum). Namely, assume the following an inequality is satisfied :

$$
f(n) \leq g(n) .
$$


Namely, comparing inside logarithm of inequalities, the following conditions are satisfied :

$$
3^{2^{n}} \leq \frac{\operatorname{rad}\left(3^{2^{n+1}}\left(3^{2^{n+1}}-1\right)\right)}{\operatorname{rad}\left(3^{2^{n}}\left(3^{2^{n}}-1\right)\right)}
$$

Hence, using inequalities( 2.12 ), the following inequalities are satisfied :

$$
3^{2^{n}} \operatorname{rad}\left(3^{2^{n}}\left(3^{2^{n}}-1\right)\right) \leq \operatorname{rad}\left(3^{2^{n+1}}\left(3^{2^{n+1}}-1\right)\right)
$$

Therefore, using inequalities( 2.12$)$,

$$
\begin{aligned}
& 3^{2^{n}} \operatorname{rad}\left(3^{2^{n}}\left(3^{2^{n}}-1\right)\right) \\
& =3^{2^{n}} 3 \operatorname{rad}\left(3^{2^{n}}-1\right) \quad\left(\because \operatorname{rad} 3^{2^{n}}=3\right) \\
& \leq 3 \operatorname{rad}\left(3^{2^{n+1}}-1\right) \quad(\because \operatorname{inequalities}(2.13)) \\
& \leq \operatorname{rad}\left(3^{2^{n}}-1\right) \operatorname{rad}\left(3^{2^{n}}+1\right) \\
& \quad\left(\because \operatorname{rad}\left(3^{2^{n+1}}-1\right) \leq \operatorname{rad}\left(3^{2^{n}}-1\right) \operatorname{rad}\left(3^{2^{n}}+1\right)\right)
\end{aligned}
$$

Therefore, the following inequality is satisfied :

$$
3^{2^{n}} \leq \operatorname{rad}\left(3^{2^{n}}+1\right)
$$

Because $\operatorname{rad}\left(3^{2^{n}}+1\right)$ is even number, the following inequalities are satisfied :

$$
3^{2^{n}} \leq \operatorname{rad}\left(3^{2^{n}}+1\right)<\frac{3^{2^{n}}+1}{2}
$$

This inequality ( 2.16$)$ is contradiction as follows :

$$
\frac{3^{2^{n}}+1}{2}<3^{2^{n}} \quad,(n>0)
$$

Therefore, the following condition is satisfied :

$$
f(n)>g(n) .
$$

Using the relation of inequality(2.18), the rate of increase in $\log \left(3^{2^{n}}\right)$ is greater than the rate of in $\log \left(\operatorname{rad}\left(3^{2^{n}}\left(3^{2^{n}}-1\right)\right)\right)$. Namely, the following conditions are satisfied :

$$
P_{K_{\epsilon}}(a, b, c)=\frac{\log (c)}{\log \left(K_{\epsilon} \operatorname{rad}(a b c)\right)}=\frac{\log \left(3^{2^{n}}\right)}{\log \left(K_{\epsilon} \operatorname{rad}\left(3^{2^{n}}\left(3^{2^{n}}-1\right)\right)\right)}>1
$$

$$
P_{K_{\epsilon}}(a, b, c) \text { is a monotone increasing, }
$$

where $a=1, b=3^{2^{n}}-1$ and $c=3^{2^{n}}$.

Note the constant $K_{\epsilon}$ can be choose 1 , that is, $K_{\epsilon}=1$.

\subsection{Proof of Theorem (1.7).}

We show that the proof of Theorem(1.7)

Proof. Let $\alpha>0$ be positive real number. Let be $\operatorname{rad}(a b c)<c$, where the positive integer $c$ is not a prime number. Because if the positive integer $c>0$ is a prime number, then $\operatorname{rad}(a b c)>c$ is satisfied. Thus, let the positive integer $c>0$ be a composite number (not a prime number).

Put positive integer $a, b$ and $c$ as follows :

$$
a=1, b=3^{2^{n}}-1, c=3^{2^{n}},(n>1)
$$


Thus the following condition is satisfied :

$$
a+b=c
$$

Using Lemma(1.6), for all real number $\epsilon>0$ and $K_{\epsilon} \geq 1$, the following conditions are satisfied :

$$
\begin{aligned}
& \log \left(3^{2^{n}}\right)>\log \left(K_{\epsilon} \operatorname{rad}\left(3^{2^{n}}\left(3^{2^{n}}-1\right)\right)\right) \quad(\because \operatorname{Lemma}(1.6)) \\
& P_{K_{\epsilon}}(a, b, c)=\frac{\log (a)}{\log \left(K_{\epsilon} \operatorname{rad}(a b c)\right)}>0, \quad(\because \operatorname{Lemma}(1.6)) \\
& R_{\alpha}^{-}(c)=\frac{\sqrt{2 \pi} \alpha}{e c-1}>0 \quad(\because \text { set } x=c \text { on Definition(1.5) })
\end{aligned}
$$

Because for sufficiently large $c>1$, the following conditions are satisfied :

$$
\begin{aligned}
& P_{K_{\epsilon}}(a, b, c) \text { is monotonically increasing. } \\
& \frac{1}{c R_{\alpha}^{-}(c)}=\frac{e}{\sqrt{2 \pi} \alpha}\left(1-\frac{1}{e c}\right)<\frac{e}{\sqrt{2 \pi} \alpha} \quad\left(=\frac{1.0844}{\alpha} \cdots\right)
\end{aligned}
$$

Therefore, using these conditions above, for all sufficiently large integer $c>1$ (that is, $n>1$ ), the following condition is satisfied :

$$
\frac{P_{K_{\epsilon}}(a, b, c)}{c R_{\alpha}^{-}(c)}>1
$$

Hence, for all sufficiently large integer $c>1$ (that is, $n>1$ ), the following conditions are satisfied :

$$
\begin{aligned}
& \left(1+\frac{1}{P_{K_{\epsilon}}(a, b, c)}\right) \\
& <\left(1+\frac{1}{P_{K_{\epsilon}}(a . b . c)}\right)^{\frac{P_{K_{\epsilon}(a, b, c)}^{c R_{\alpha}^{-}(c)}}{1}}(\because \text { inequaulity }(2.28)) \\
& =\left(\left(1+\frac{1}{P_{K_{\epsilon}}(a, b, c)}\right)^{P_{K_{\epsilon}}(a, b, c)}\right)^{\frac{1}{c R_{\alpha}^{-}(c)}} \\
& \leq \lim _{c \rightarrow \infty}\left(\left(1+\frac{1}{P_{K_{\epsilon}}(a, b, c)}\right)^{P_{K_{\epsilon}}(a, b, c)}\right)^{\frac{1}{c R_{\alpha}^{-}(c)}} \\
& \left(\because P_{K_{\epsilon}}(a, b, c) \text { is monotonically increase by Lemma }(1.6)\right) \\
& \leq \exp \left(\frac{1}{c R_{\alpha}^{-}(c)}\right) \\
& \left(\because \lim _{c \rightarrow \infty}\left(1+\frac{1}{P_{K_{\epsilon}}(a, b, c)}\right)^{P_{K_{\epsilon}}(a, b, c)}=\exp (1)\right)
\end{aligned}
$$

Namely, the following inequality is satisfied :

$$
\left(1+\frac{1}{P_{K_{\epsilon}}(a, b, c)}\right) \leq \exp \left(\frac{1}{c R_{\alpha}^{-}(c)}\right)
$$

Transforming inequalities the above and definition of $P_{K_{\epsilon}}(a, b, c)$, the following conditions are satisfied :

$$
\begin{aligned}
\frac{\log \left(K_{\epsilon} \operatorname{rad}(a b c)\right)}{\log (c)} & =\frac{1}{P_{K_{\epsilon}}(a, b, c)} \\
& <\exp \left(\frac{1}{c R_{\alpha}^{-}(c)}\right)-1
\end{aligned}
$$


Therefore, the following inequalities are satisfied :

$$
\begin{aligned}
\log \left(K_{\epsilon} \operatorname{rad}(a b c)\right) & <\left(\exp \left(\frac{1}{c R_{\alpha}^{-}(c)}\right)-1\right) \log (c) \\
& =\log (c)\left(\exp \left(\frac{1}{c R_{\alpha}^{-}(c)}\right)-1\right) \\
& =\log (c)\left(\exp \left(\frac{e}{\sqrt{2 \pi} \alpha}\left(1-\frac{1}{c c}\right)\right)-1\right)
\end{aligned}
$$

Therefore, for all sufficiently large positive integer $c>1$ (that is, $n>1$ ), the following inequality is satisfied :

$$
\log \left(K_{\epsilon} \operatorname{rad}(a b c)\right)<\log (c)^{\left(\exp \left(\frac{e}{\sqrt{2 \pi} \alpha}\right)-1\right)}
$$

Comparing in logarithm of above inequality, the following inequality is satisfied :

$$
K_{\epsilon} \operatorname{rad}(a b c)<c^{\left(\exp \left(\frac{e}{\sqrt{2 \pi \alpha}}\right)-1\right)}
$$

\subsection{Proof of Theorem (1.8).}

Next, we show that the negation of the weak abc conjecture is satisfied as follows :

Proof. for positive real numbers $\epsilon>0$ and $\alpha>0$, set as follows:

$$
\alpha=\frac{e}{\sqrt{2 \pi} \log \left(\frac{\epsilon+2}{\epsilon+1}\right)}
$$

Namely, the exponet of $c$ on the inequality ( 1.15 ) in theorem(1.7) set as follows

$$
\frac{1}{1+\epsilon}=\exp \left(\frac{e}{\sqrt{2 \pi} \alpha}\right)-1
$$

Hence, the following conditions are satisfied :

$$
\exp \left(\frac{e}{\sqrt{2 \pi} \alpha}\right)=\frac{\epsilon+2}{\epsilon+1}>1
$$

Therefore, the following condition is satisfied :

$$
\alpha=\frac{e}{\sqrt{2 \pi} \log \left(\frac{\epsilon+2}{\epsilon+1}\right)}
$$

Because the inequalities $\epsilon>0$ and $(\epsilon+2) /(\epsilon+1)>1$ are satisfied, thus the positive real number $\epsilon>0$ is satisfied as follows :

$$
\log \left(\frac{\epsilon+2}{\epsilon+1}\right)>0
$$

Thus, the following conditions also is satisfied :

$$
\alpha=\frac{e}{\sqrt{2 \pi} \log \left(\frac{\epsilon+2}{\epsilon+1}\right)}>0
$$

Therefore, using the discussion above :

$$
K_{\epsilon} \operatorname{rad}(a b c)<c^{\frac{1}{1+\epsilon}}
$$


where

$$
\begin{aligned}
& \epsilon=\frac{1}{\exp \left(\frac{e}{\sqrt{2 \pi} \alpha}\right)-1}-1>0 \\
& \alpha>\frac{e}{\sqrt{2 \pi} \log (2)}>0
\end{aligned}
$$

For all $\epsilon>0$ set as $\bar{K}_{\epsilon}:=K_{\epsilon}{ }^{1+\epsilon}$. Therefore For all $\epsilon>0 \bar{K}_{\epsilon}=K_{\epsilon}{ }^{1+\epsilon} \geq 1, n>1$, there exists triples $(a, b, c)$ of coprime integers such that

$$
\begin{aligned}
& a=1, \\
& b=3^{2^{n}}-1, \\
& c=3^{2^{n}},(n>1) \\
& a+b=c .
\end{aligned}
$$

The following conditions are satisfied :

$$
\bar{K}_{\epsilon} \operatorname{rad}(a b c)^{1+\epsilon}<c .
$$

After all, there exists countable infinite triples of coprime integers such that satisfies the weak abc conjecture. Namely, the weak abc conjecture is not true.

\section{Conclisions.}

We derive that the negation of the weak abc conjecture using the functions $R_{\alpha}^{-}(x)$. Therefore, we showed that the weak abc conjecture is not true. Furthermore, the strong abc conjecture is not true.

We started this paper to the introduction of the functions $R_{\alpha}^{-}(x)$. We obtained the functions $R_{\alpha}^{-}(x)$ using the concept of Statistical Mechanics. We think that Number theory and Statistical Mechanics are considered to be closely related.

\section{Acknowledgments.}

We would like to thank all the people who supported this challenge and to express my deepest respect for giving us the idea. We would like to explain how to derive functions used in this paper if it gives us an opportunity,

\section{REFERENCES}

[1] Wladyslaw Narkiewicz, The Development of Prime Number Theory, Springer, 2000

[2] Feynman et.al, The Feynman, Lecture on Physics, Volume III, Quantum Mechanics ,1963

[3] Michel Waldschmidt, On the abc Conjecture and some of its consequences,Université P. et M. Curie (Paris VI), 2018

[4] Michel Waldschmidt, Lecture on the abc conjecture and some of its consequences, Springer Proceedings in Mathematics and Statistics 98 , 211-230.,(2015)

[5] Deriving Von Koch's inequality without using the Riemann Hypothesis, Seiji Fujino, Preprints 2021, 2021120074 (doi: 10.20944/preprints202112.0074.v1).

[6] Seiji Fujino, Expansion of Planck's law and Application to Number Theory, (In preparation) 